\title{
Cost-effectiveness analysis of online hemodiafiltration versus high-flux hemodialysis
}

\section{Francesco Ramponi ${ }^{1,2}$ \\ Claudio Ronco ${ }^{1,3}$ \\ Giacomo Mason' \\ Enrico Rettore ${ }^{4}$ \\ Daniele Marcelli ${ }^{5,6}$ \\ Francesca Martino 1,3 \\ Mauro Neri ${ }^{1,7}$ \\ Alejandro Martin-Malo ${ }^{8}$ \\ Bernard Canaud ${ }^{5,9}$ \\ Francesco Locatelli ${ }^{10}$}

International Renal Research Institute (IRRIV), San Bortolo Hospital, Vicenza, ${ }^{2}$ Department of Economics and Management, University of Padova,

Padova, ${ }^{3}$ Department of Nephrology,

San Bortolo Hospital, Vicenza,

${ }^{4}$ Department of Sociology and Social

Research, University of Trento, FBK-

IRVAPP \& IZA, Trento, Italy; '5urope,

Middle East, Africa and Latin America

Medical Board, Fresenius Medical

Care,, Bad Homburg, Germany;

${ }^{6}$ Danube University, Krems, Austria;

${ }^{7}$ Department of Management and

Engineering, University of Padova,

Vicenza, Italy; ${ }^{8} \mathrm{Nephrology}$ Unit,

Reina Sofia University Hospital,

Córdoba, Spain; ' $\mathrm{School}$ of Medicine, Montpellier University, Montpellier,

France; ${ }^{10}$ Department of Nephrology,

Manzoni Hospital, Lecco, Italy

Correspondence: Francesco Ramponi International Renal Research Institute Vicenza (IRRIV), San Bortolo Hospital, Viale Rodolfi 37, 36 100 Vicenza, Italy Tel +390444753949

Email francesco.ramponi@hotmail.com
This article was published in the following Dove Press journal:

ClinicoEconomics and Outcomes Research

22 September 2016

Number of times this article has been viewed

Background: Clinical studies suggest that hemodiafiltration (HDF) may lead to better clinical outcomes than high-flux hemodialysis (HF-HD), but concerns have been raised about the cost-effectiveness of HDF versus HF-HD. Aim of this study was to investigate whether clinical benefits, in terms of longer survival and better health-related quality of life, are worth the possibly higher costs of HDF compared to HF-HD.

Methods: The analysis comprised a simulation based on the combined results of previous published studies, with the following steps: 1) estimation of the survival function of HF-HD patients from a clinical trial and of HDF patients using the risk reduction estimated in a metaanalysis; 2) simulation of the survival of the same sample of patients as if allocated to HF-HD or HDF using three-state Markov models; and 3) application of state-specific health-related quality of life coefficients and differential costs derived from the literature. Several Monte Carlo simulations were performed, including simulations for patients with different risk profiles, for example, by age (patients aged 40, 50, and 60 years), sex, and diabetic status. Scatter plots of simulations in the cost-effectiveness plane were produced, incremental cost-effectiveness ratios were estimated, and cost-effectiveness acceptability curves were computed.

Results: An incremental cost-effectiveness ratio of $€ 6,982$ /quality-adjusted life years (QALY) was estimated for the baseline cohort of 50-year-old male patients. Given the commonly accepted threshold of $€ 40,000 / \mathrm{Q} A L Y, H D F$ is cost-effective. The probabilistic sensitivity analysis showed that HDF is cost-effective with a probability of $\sim 81 \%$ at a threshold of $€ 40,000 / \mathrm{QALY}$. It is fundamental to measure the outcome also in terms of quality of life. HDF is more cost-effective for younger patients.

Conclusion: HDF can be considered cost-effective compared to HF-HD.

Keywords: cost-effectiveness, hemodialysis, hemodiafiltration

\section{Introduction}

Renal replacement treatment, in the form of kidney transplantation or dialysis, is a life-supporting therapy for patients with end-stage renal disease. At the end of 2010, $\sim 1.81$ million chronic kidney disease patients globally were undergoing hemodialysis (HD) treatment, with an estimated 90,000 (5\%) thereof being treated with hemodiafiltration (HDF). ${ }^{1}$ Online HDF has been suggested as a possible cost-effective alternative compared to standard HD, mainly due to its superior middle molecule clearance resulting from the combination of diffusion and convection solute removal mechanisms. Several studies suggest that HDF may lead to better clinical results than high-flux HD (HF-HD). ${ }^{2,3}$ According to a recent survey, HDF was started to prevent 
dialysis complications, increase the efficiency of dialysis for uremic accumulates, treat dialysis-related hypotension and dialysis-related amyloidosis, and for intractable pruritus, restless leg syndrome and arthralgia not related to dialysis-related amyloidosis. ${ }^{4}$ However, to date, randomized studies have not conclusively supported the superiority of HDF regarding clinical outcomes. ${ }^{5-7}$ In addition, concerns have been raised about its cost-utility, ${ }^{8}$ leading investigators to conduct cost analyses to assess the differential expenditures between the two therapies. ${ }^{9}$ In a recent editorial, Vanholder et al concluded that more expensive therapies should be reimbursed only when evidence of their cost-utility, defined as the ratio of treatment cost to treatment outcome, taking into consideration also the quality of life (QoL), is sufficiently robust. ${ }^{10}$ The cost of HDF per se has decreased in recent years due to 1) increased market penetration and the subsequent economy of scale in the production of equipment and disposables, 2 ) the pressure on health care costs due to the economic crises prevailing since 2008, and 3 ) the safety and reliability of online method that have reduced demand for microbiological testing and monitoring. ${ }^{11,12}$ Furthermore, after the publication of the study of Canaud et al, ${ }^{2}$ HDF treatment effectiveness has increasingly been understood to be positively associated with the magnitude of the convection volume used, resulting in trends toward the application of higher HDF convection volumes than were applied in earlier cost analyses. Accordingly, there is a need for a new cost-effectiveness evaluation. The aim of this study is to assess the cost-effectiveness of HDF compared to HD by a simulation using existing study data. These data facilitate an understanding of whether longer survival and better health-related quality of life (HRQoL) are able to offset the possibly higher costs of HDF, and thus whether it is worth investing in this more innovative therapy.

\section{Methods}

The analysis comprised a simulation ${ }^{13-15}$ based on results from combined previous observational studies, randomized clinical trials, and a meta-analysis with the following steps: 1) estimation of a) the survival function of HF-HD patients from the Membrane Permeability Outcome Study ${ }^{16}$ dataset and $b$ ) estimation of the survival function of HDF patients using the risk reduction estimates due to the treatment effect from the meta-analysis of Mostovaya et al ${ }^{17}$ (even though it includes also studies comparing HDF to low-flux HD, it was considered the best proxy with respect to other alternative meta-analyses available in the literature); 2) simulation of the survival of the same sample of patients as if allocated to HF-HD or HDF using three-state Markov models; 3) application of state-specific HRQoL coefficients and differential costs (materials, testing, and consumption of water) derived from the literature. In addition, in a secondary subgroup analysis we considered the $\mathrm{ESHOL}^{5}$ estimations related to diabetics and nondiabetics. This was done because among all the studies considered the ESHOL study provides results related to these particular subgroups of patients. Two mirror-image Markov models (Figure 1) were developed with the aim to generate a simulation comparing the survival of the same sample of patients treated with HF-HD and HDF. Three possible states were considered: alive and under therapy, dead due to the disease under therapy, and dead for other cause (ie, considering the overall mortality, based on age- and sex-adjusted life tables).

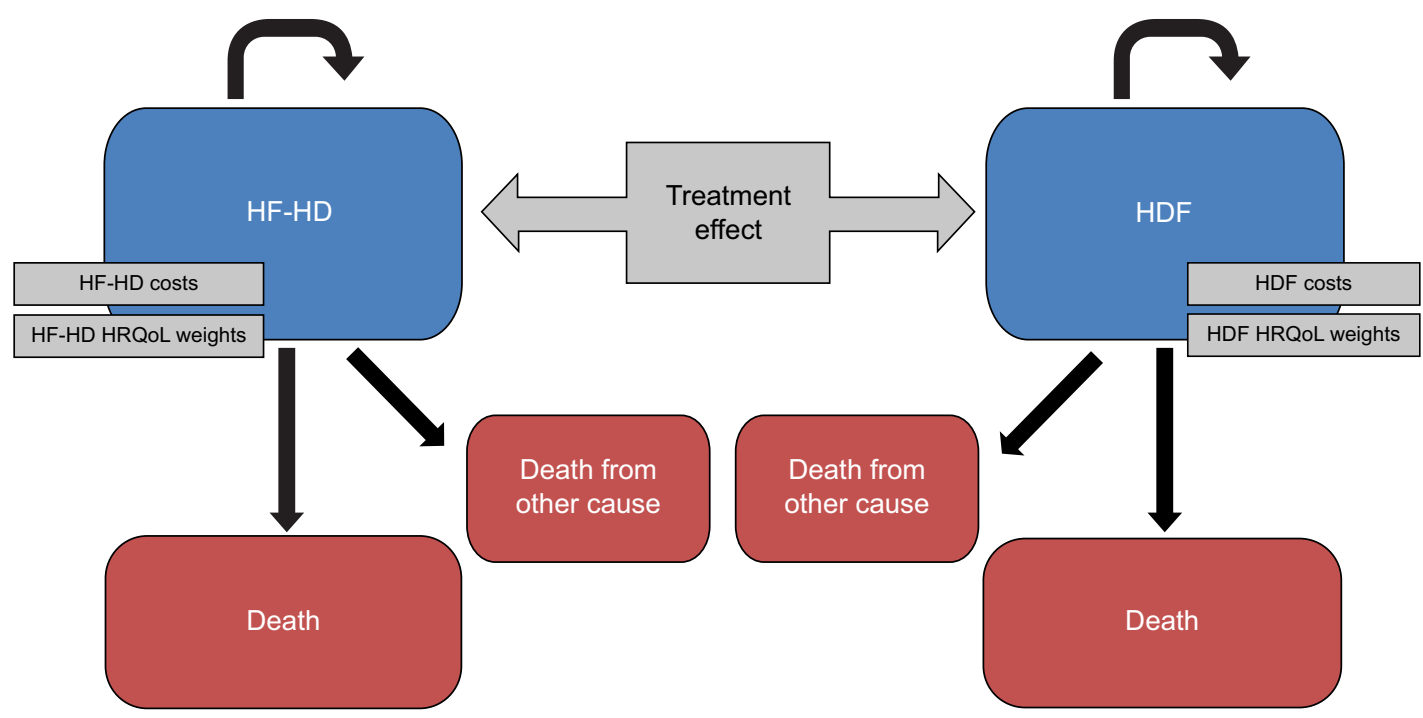

Figure I Structure of the Markov model.

Abbreviations: HDF, hemodiafiltration; HF-HD, high-flux hemodialysis; HRQoL, health-related quality of life. 
The probabilities defining the transition of patients between each of these states were calculated from the previously estimated hazard functions and were specified over a defined time frame (cycle). The model was run for a specified number of cycles to see how the hypothetical cohort of patients moved between states. The length of the cycle was fixed to 1 year and the number of cycles to ten, for a total of 10 years. Each state of the model was associated with a HRQoL coefficient and a cost. The costs were considered from a societal perspective. Accordingly, total dialysis costs can be divided into: 1) direct health care costs, such as staff, material, vascular access, routine diagnostics, hospitalization, drugs, and medications; 2) direct nonhealth care costs, such as cost of transport and cost of informal care; and 3) indirect nonhealth care costs, productivity losses due to disability or premature death. It is reasonable to assume that direct nonhealth care costs and indirect nonhealth care costs are the same for the two therapies, and also that most of the direct health care costs do not vary by treatment. As a consequence, the comparison on the incremental costs of HDF with respect to HF-HD focused only on the costs of equipment, disposables, ultrapure water testing, and water consumption. ${ }^{18}$ In order to obtain more generalizable results, we analyzed two alternative cost settings, involving or not involving the use of ultrapure water. The first was based on the assumption that, since dialyzers used to perform both HDF and HF-HD are designed to have better solute clearances on a wider range of uremic toxins and higher hydraulic permeability, it is reasonable to argue that the same water and dialysis fluid microbiological purity were necessary for both therapies due to backfiltration phenomena, leading to similar costs of water testing. As a consequence, and because differences in total water consumption are negligible, it was possible to limit the analysis to the costs of disposables (blood lines, dialyzers) and equipment only. In the prospective observational study by Oates et al, ${ }^{9}$ some patients on HF-HD were switched to HDF and others remained on HF-HD. They were then prospectively followed for 12 months. The additional cost of disposables related to HDF was of $€ 1.32$ per session ( $€ 207$ per annum [p.a.]) in the standard case, that is, with the use of a cuvette for relative blood volume monitoring. The alternative cost setting was based on the analysis by Lebourg et $\mathrm{al}^{12}$ where, in addition to a cost variability due to disposables and monitors, the difference in cost between the two different therapies caused by water analysis and water consumption was considered. The additional HDF cost per session ranged from a minimum value of $-€ 1.29$ ( $€ 202$ p.a.) to a maximum value of $€ 4.86$
(€730 p.a.). Once the relevant costs were determined, the corresponding estimated and discounted costs (or a differential cost) taken from the literature were allocated to each state of the model.

Contradicting results have been reported on the difference in QoL related to HF-HD and HDF. Few studies addressed the QoL evaluation, and none provided HRQoL coefficients for the specific comparison of HF-HD and HDF, fundamental for the purpose of this analysis. Mazairac et $\mathrm{al}^{8}$ estimated higher scores with Euroqol 5D for patients on HDF compared with HD. This preference-based measure yields a set of weights on which quality-adjusted life years (QALYs) calculations can be based. In this study, the comparison was made between HDF and low-flux HD. As there are no other data about QoL differences between HDF and HF-HD in the literature, these coefficients have been used in our simulation. In particular, the HRQoL coefficients linked to age were selected for the model because otherwise a fundamental component of the benefit measurement would have been neglected.

As recommended by the economic analysis guidelines, ${ }^{19}$ an annual discount rate of 3.5\% for both costs and benefits was selected, and changes in results as a function of different values of these parameters were considered. Parameter uncertainty was included in the model through a probabilistic sensitivity analysis, taking the intrinsic imprecision of the estimate of the parameters of the survival model into account. Consequently, the quality and quantity of information available can be reflected in the probability distributions assigned to each input parameter in the model. ${ }^{20}$ Several Monte Carlo simulations were performed, also for patients with different risk profiles by age (patients aged 40,50, and 60 years), sex, and diabetic status. ${ }^{21,22}$ For the sake of simplicity, the cohort of simulated 50-year-old male patients were selected as the reference population for the analysis. As discussed in the next section, we found similar results in all age subgroups, with identical conclusions in terms of cost-effectiveness; therefore, we used this cohort to briefly recap the main results of our analysis. The characteristics of this cohort are coherent with the results from the EDTA registry reported by van de Luijtgaarden et al: $:^{23}$ in the last 20 years indeed the mean age of patients who started with HD treatment was $55-60$ years, and on average $>60 \%$ of patients were male.

Output analyses included scatter plots of simulations in the cost-effectiveness plane, the estimation of incremental cost-effectiveness ratios (ICERs) and the computation of cost-effectiveness acceptability curves (CEACs). 


\section{Results}

The results of the 1,000 Monte Carlo simulations for the cohort of 50-year-old male patients are shown in the cost-effectiveness scatter plot in Figure 2. From the mean values of all the simulations in this specific cohort of patients, the ICER was €6,982/ QALY. On the basis of these simulations it was possible to derive the probability of cost-effectiveness given different thresholds, as shown in Figure 3. The CEAC corresponding to HDF starts from 0, meaning that there is no possibility that this alternative therapy is cost-saving. The threshold must be at least $€ 2,000$ per QALY to have a probability of cost-effectiveness $>0$. The probability of cost-effectiveness increased to $50 \%$ with a threshold of $€ 7,000 / \mathrm{QALY}$, to $70 \%$ with a threshold of $€ 15,000$ / QALY and to $81 \%$ with the commonly accepted threshold of $€ 40,000 / \mathrm{QALY}$. The probability of cost-effectiveness always stayed below $84 \%$ for a threshold of over $€ 40,000 / Q A L Y$. This asymptotic value results from the fact that a fraction of the simulations represent cases where the alternative therapy (HDF) causes higher costs and provided fewer benefits. This means that even with any budget constraint, there is some probability that the alternative therapy is not cost-effective and the traditional one (HF-HD) is preferred.

The same analyses were carried out for the subgroups of 40-, 50-, and 60-year-old male and female patients

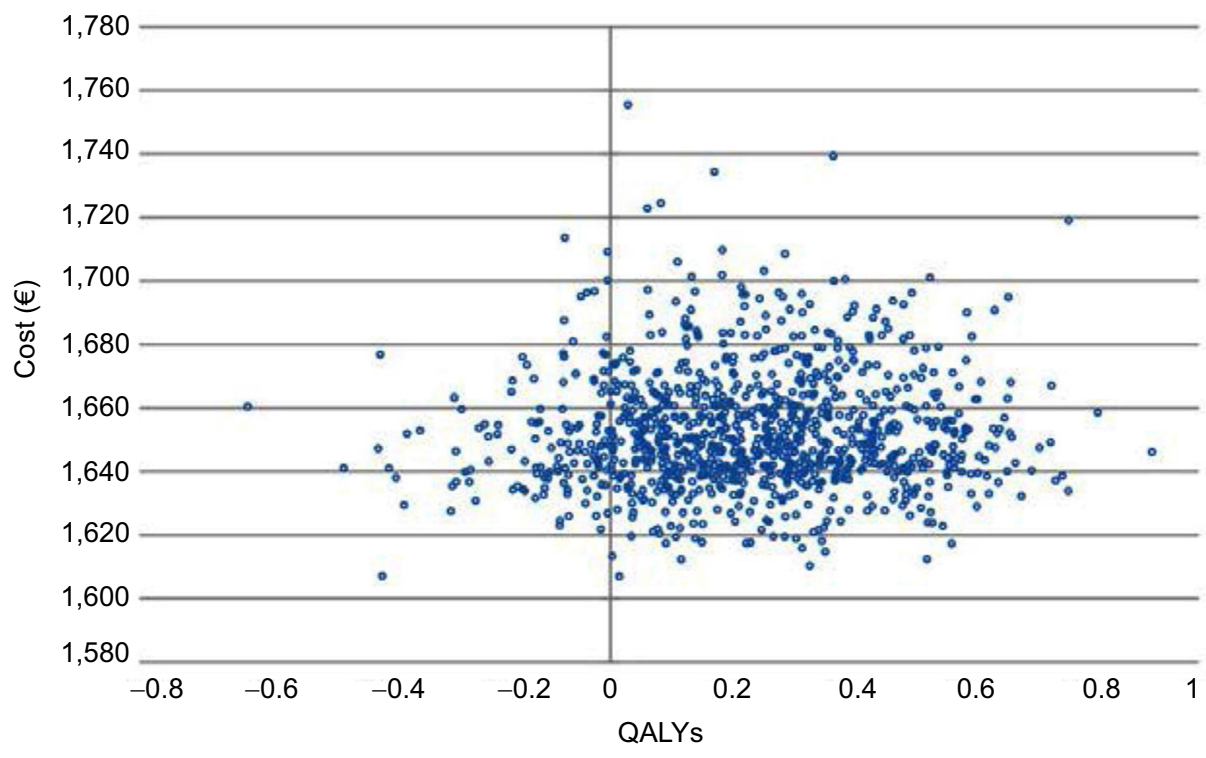

Figure 2 Cost-effectiveness plane for 50-year-old male patients. Abbreviation: QALYs, quality-adjusted life years.

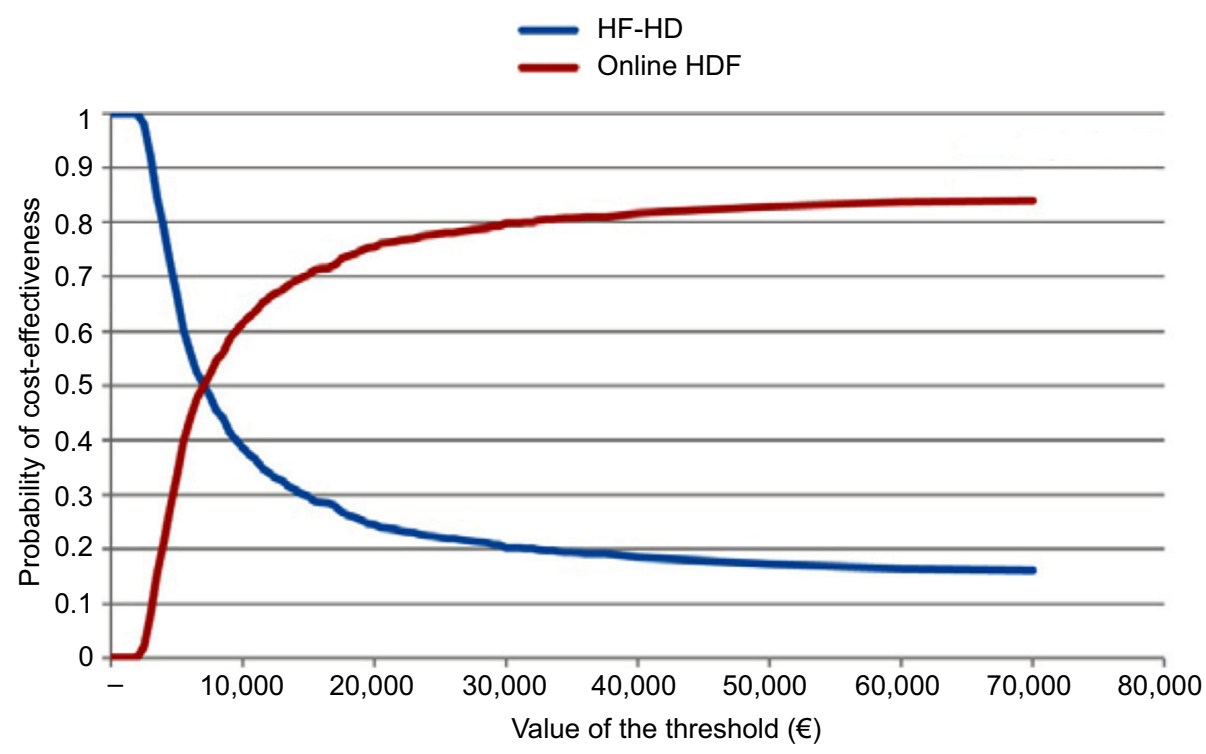

Figure 3 Cost-effectiveness acceptability curves for male patients aged 50 years old treated with high-flux HD or online HDF. Abbreviations: HDF, hemodiafiltration; HF-HD, high-flux hemodialysis. 
(Table 1): HDF appears to be more cost-effective for younger patients. This message is reinforced in Figure 4, which shows that a given threshold value is associated to a higher probability of HDF being cost-effective for the 40- and 50-yearold patients than for the 60 -year-old ones. Moreover, for the 60 -year-old groups there is a probability of around $28 \%$ for both females and males that HDF is not cost-effective even at extreme values of the threshold.

Regarding the discount rates for costs and benefits, results were robust even after considering alternative scenarios with lower or higher discount rates. By applying a $0 \%$ or a $5 \%$ discount rate for both costs and effects, 50-year-old male patient ICER decreased to $€ 6,676 / \mathrm{QALY}$ and increased to $€ 6,960 /$ QALY, respectively, confirming the robustness of the estimations.

A further analysis was performed to investigate the importance of the qualitative component of the QALYs (QoL)

Table I Incremental costs and QALYs for subgroups of patients

\begin{tabular}{|c|c|c|c|c|c|c|}
\hline & \multicolumn{3}{|c|}{ Males, age, years } & \multicolumn{3}{|c|}{ Females, age, years } \\
\hline & 40 & 50 & 60 & 40 & 50 & 60 \\
\hline Incremental cost $(€)$ & 1,679 & $\mathrm{I}, 653$ & $\mathrm{I}, 538$ & $\mathrm{I}, 707$ & $\mathrm{I}, 70 \mathrm{I}$ & $I, 64 I$ \\
\hline Incremental QALYs & 0.293 & 0.237 & 0.112 & 0.290 & 0.248 & 0.120 \\
\hline ICER (€/QALY) & 5,732 & 6,982 & 13,668 & 5,878 & 6,872 & 13,697 \\
\hline
\end{tabular}

Abbreviations: ICER, incremental cost-effectiveness ratio; QALYs, quality-adjusted life years. compared to the quantitative one (life years). The effect of using overall HRQoL coefficients (rather than the HRQoL coefficients linked to patient age) on the cohort of 50-year-old male patients is shown in Figure 5. The mean ICER increased to $€ 19,423 /$ QALY but, more importantly, almost $37 \%$ of the simulations were on the left of the QALY zero value (less effectiveness, more costs) and, accordingly, the uncertainty around the decision whether to invest in the new therapy was much higher. Even an infinite value of the threshold would not lead to a probability of cost-effectiveness $>65 \%$ (data not shown). This is caused by the uncertain values of the overall HRQoL coefficients, leading to some cases where one period spent under HF-HD is worth even more than the same period under HDF. This shows that it is crucial to consider the role of QoL is in such evaluations.

Using the alternative cost setting, the model becomes fully probabilistic. Previously, cost inputs were point estimates, whereas now costs vary over the range assessed by Lebourg et al. ${ }^{12}$ The CEAC for the subgroup of 50-year-old male patients shows that $21.5 \%$ of the simulations are cost-saving (Figure 6). The probability of being cost-effective reaches $80 \%$ at the commonly accepted threshold of $€ 40,000 / Q A L Y$, and it increases only by 2.4 percentage points even with a threshold of $€ 70,000 / \mathrm{QALY}$. Results are consistent with the previous cost

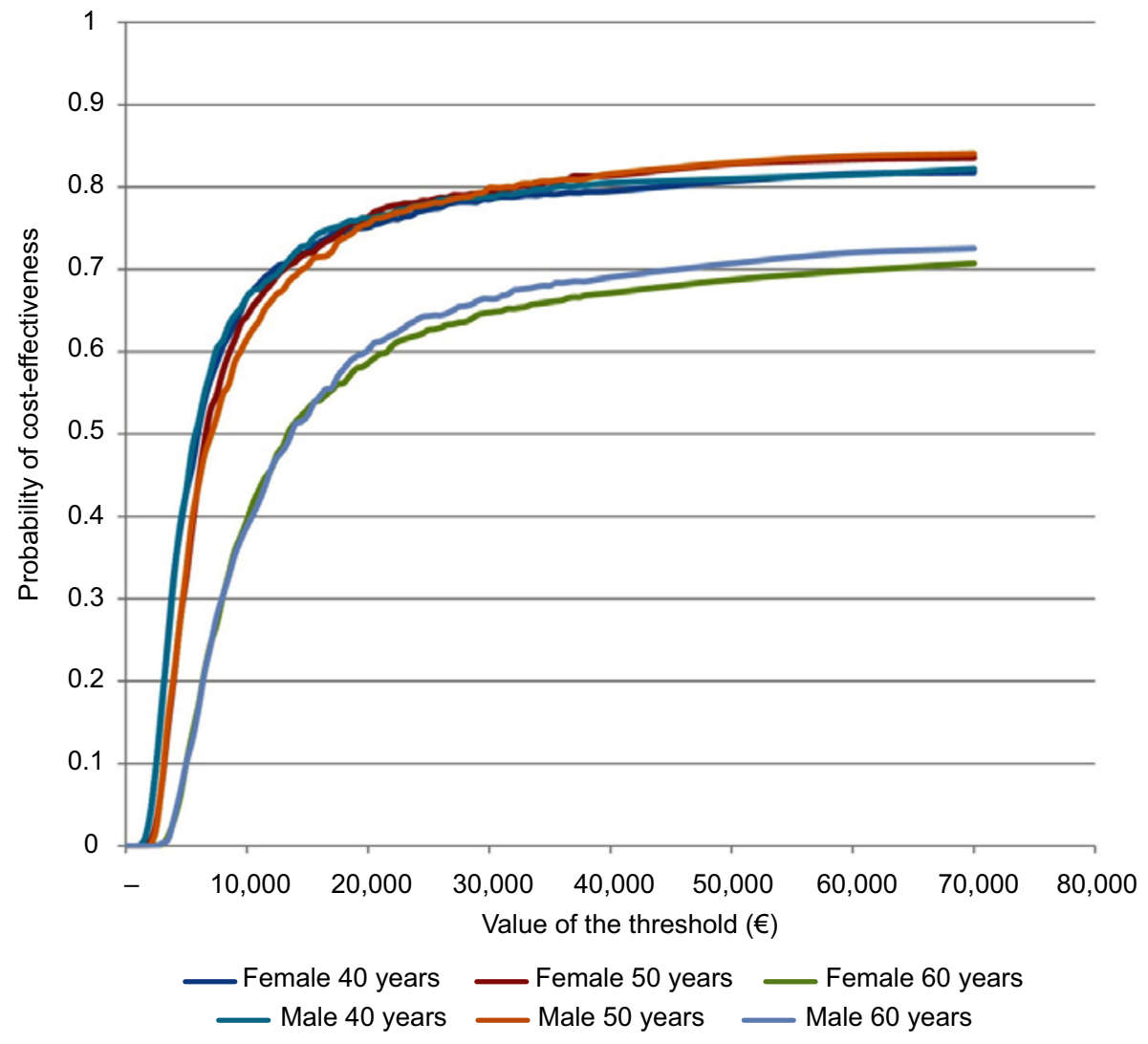

Figure 4 Cost-effectiveness acceptability curves for female and male patients aged 40, 50, and 60 years on hemodiafiltration. 


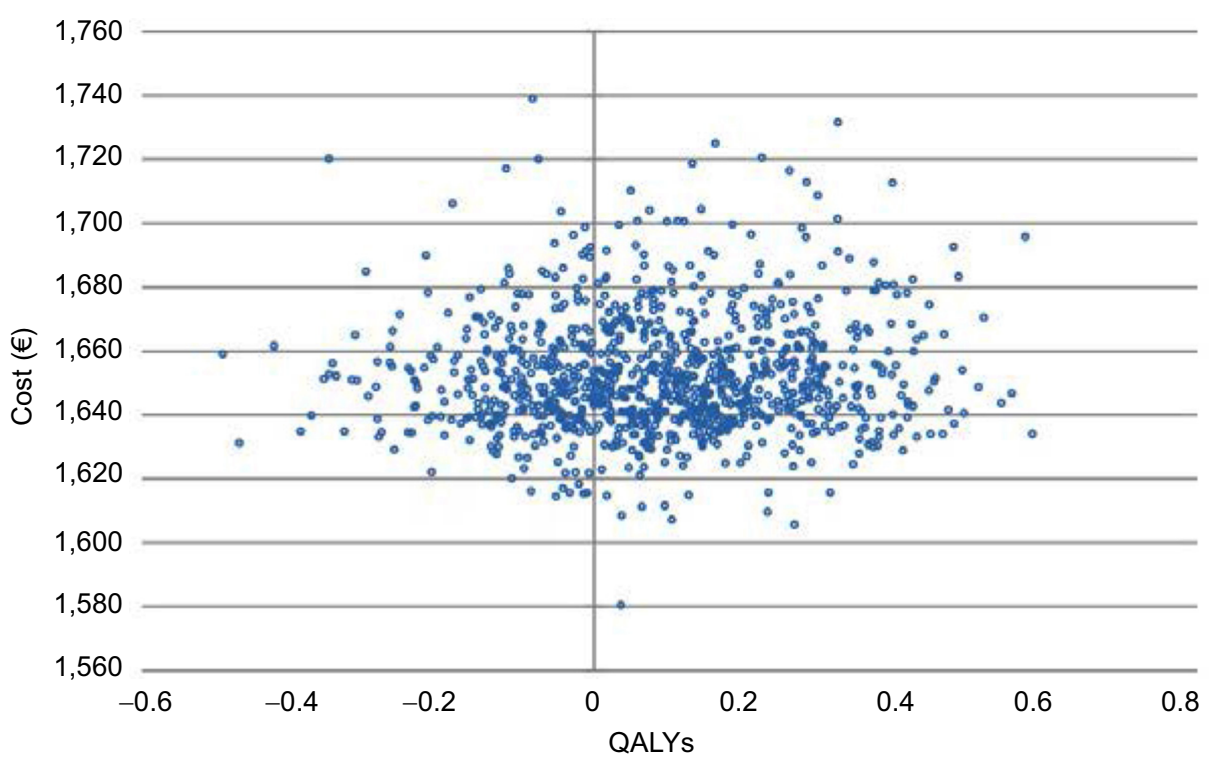

Figure 5 Cost-effectiveness plane for 50-year-old male patients, using overall health-related quality of life coefficients. Abbreviation: QALYs, quality-adjusted life years.

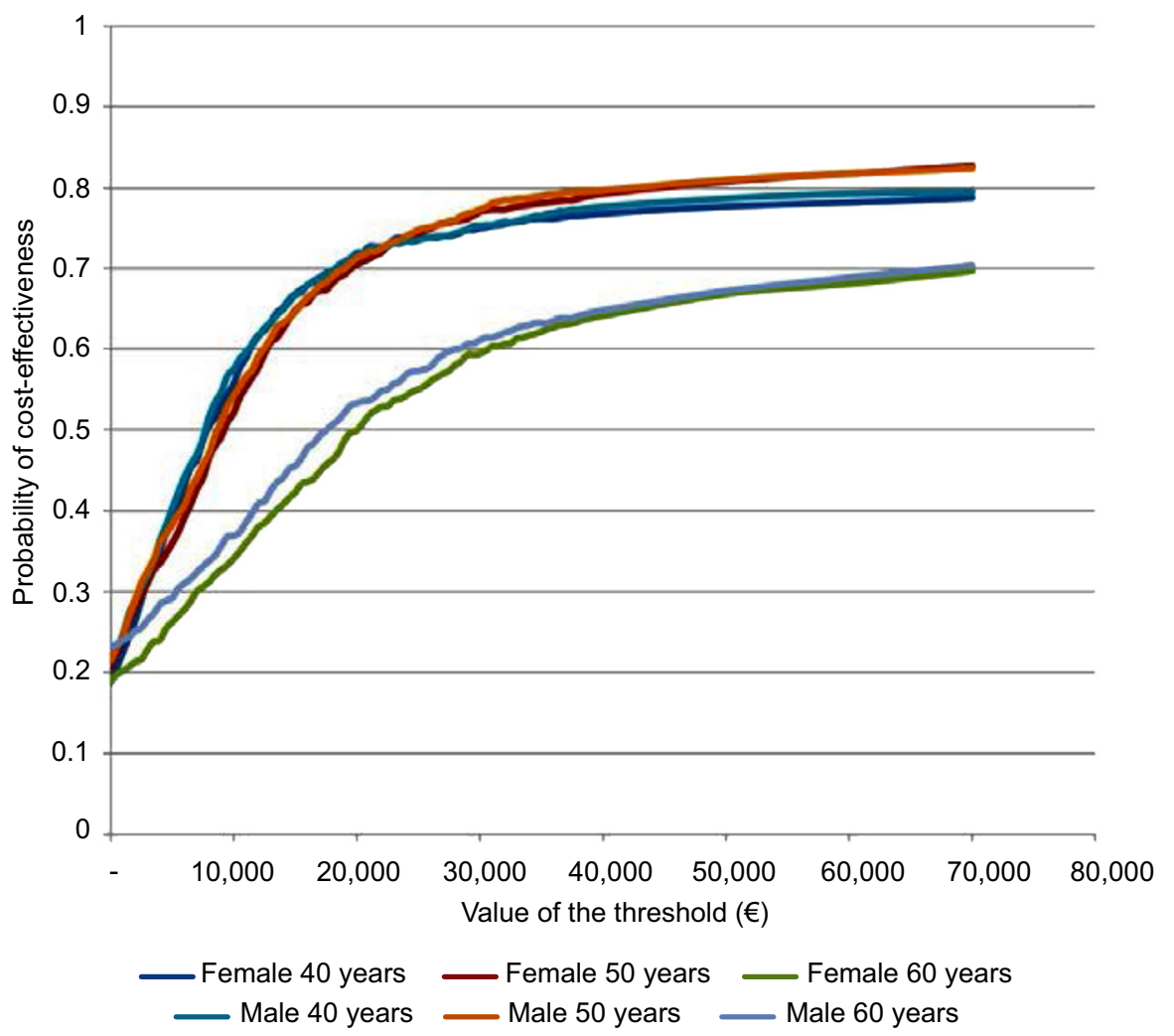

Figure 6 Cost-effectiveness acceptability curves for female and male patients aged 40, 50, and 60 years on hemodiafiltration (alternative cost setting).

setting. With regard to the mean ICERs, HDF seems a little more expensive: for instance, the ICERs of the younger patients rise from $€ 5,878 / \mathrm{QALY}$ and $€ 5,732 / \mathrm{QALY}$ to $€ 7,748 / \mathrm{QALY}$ and $€ 7,724 / \mathrm{QALY}$ for female and male patients, respectively (Table 2). This subgroup analysis confirmed the previous results: the ICER increases with the age of the cohort.
Diabetic patients follow a different survival function and are subject to a different treatment effects of the alternative therapy compared to nondiabetic patients. Diabetics are characterized by a higher mortality risk and a lower treatment effect. Nevertheless, the comparison between a cohort of 50 -year-old diabetic male patients and a cohort of 50-year-old 
Table 2 Incremental costs and QALYs for subgroups of patients (alternative cost setting)

\begin{tabular}{|c|c|c|c|c|c|c|}
\hline & \multicolumn{3}{|c|}{ Males } & \multicolumn{3}{|c|}{ Females } \\
\hline & 40 & 50 & 60 & 40 & 50 & 60 \\
\hline Incremental cost $(€)$ & 2,062 & 2,097 & 1,927 & 2,226 & 2,213 & 2,183 \\
\hline Incremental QALYs & 0.267 & 0.238 & 0.106 & 0.287 & 0.245 & 0.110 \\
\hline ICER (€/QALY) & 7,724 & 8,825 & 18,206 & 7,748 & 9,050 & 19,880 \\
\hline
\end{tabular}

Abbreviations: ICER, incremental cost-effectiveness ratio; QALYs, quality-adjusted life years.

nondiabetic male patients did not reveal significant differences (Figure 7).

\section{Discussion}

Given the commonly accepted threshold of $€ 40,000 / \mathrm{Q} A L Y$, this study demonstrated that, compared to HF-HD, HDF is a cost-effective treatment for patients on dialysis. This is particularly true for patients $<60$ years, irrespective of sex or diabetic status. Either way, the variability in the results due to different characteristics of the patients does not affect the conclusions about the cost-effectiveness of HDF. These results are quite different from those reported by Mazairac et $\mathrm{al}^{8}$ in their cost-utility analysis of HDF versus conventional low-flux HD based on the unadjusted results for convective volume of the CONvective TRAnsport STudy (CONTRAST). ${ }^{7}$ Their estimated incremental cost per quality-adjusted life year of HDF compared to low-flux HD was $€ 287,679$ and certainly not below $€ 140,000$ under the most favorable assumptions (eg, applying a convection volume $>20.3 \mathrm{~L}$ ). They argued that the HDF is characterized by higher cost and better health and was not cost-effective given the currently accepted cost-utility thresholds. However, it has to be mentioned that the same analysis performed on patients treated in Montreal (Canada) according to the same protocol yielded different, positive, results. ${ }^{24}$ Our analysis, on the other hand, reports that HDF is cost-effective with a probability of $\sim 81 \%$. This conclusion is based on what is considered the commonly accepted threshold for health policy decisions, ${ }^{25}$ which is $\sim € 40,000$ (or 30,000 GBP, or 50,000 USD) per QALY. Even if this threshold is difficult to quantify and is often based on a rule of thumb, it is commonly applied as an estimate of the forgone health resulting from services displaced to accommodate the additional costs of the new technology. Of note, the magnitude of the difference between the two studies may be related to

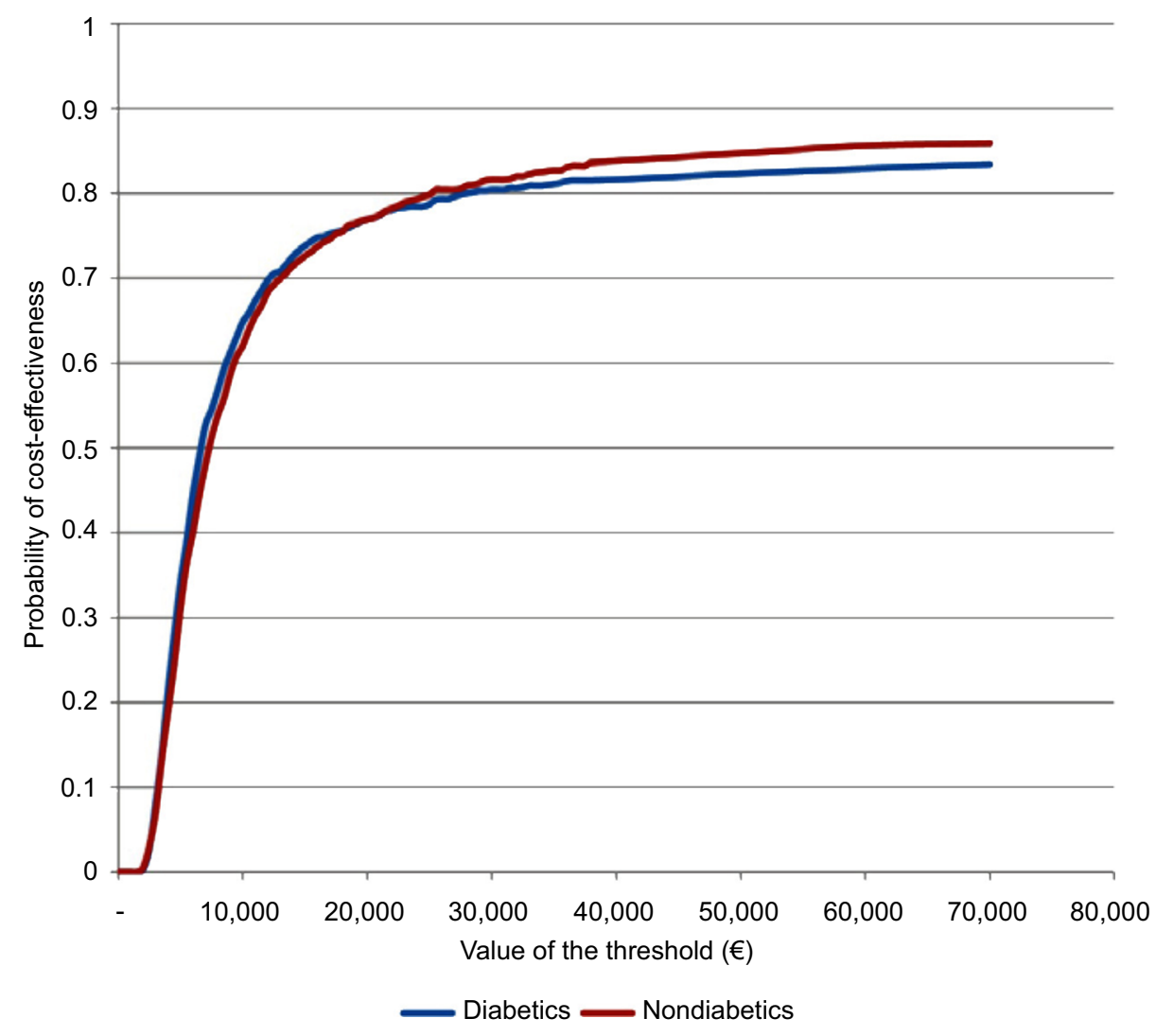

Figure 7 Cost-effectiveness acceptability curves for diabetics and nondiabetics. 
the different comparison groups, which is low-flux HD in the CONTRAST study and HF-HD in our model.

The ICER estimates show a good value for money, but some doubts about the cost-effectiveness of HDF over HF-HD remain due to the residual $\sim 20 \%$ probability of costs being higher and/or benefits being lower with HDF. This line of reasoning is also shared by McBrien and Manns ${ }^{26}$ in their commentary of the Mazairac study, where they argued that HDF is characterized by higher procedural costs and has no proven health benefit. Our study specifies that the survival difference is not the only important factor (especially considering the lack of agreement on this aspect), but that differences in terms of QoL are of fundamental importance and must be measured. This was the rationale for conducting a value-based study incorporating $\mathrm{HRQ}$ LL as a main outcome of HDF versus HF-HD. Convective treatments as HDF indeed reduce intradialytic hypotension (which affects QoL), and possibly limit the poor appetite and nutritional state in patients with chronic kidney disease. However, we believe that more research is warranted to assess the impact of HDF specifically on the nutritional state of end-stage renal disease patients. Therefore, this study recommends prioritizing the research which evaluates the QoL related to HDF.

It must be stressed that the purpose itself of an economic decision model is to synthesize all the relevant evidence in order to make a sense of it and inform the decision about the adoption of a new technology in an uncertain context. ${ }^{15}$ We have indeed identified the relevant studies involving the therapies under assessment, and we have included all the uncertainty in our parameter estimates, without neglecting any relevant information in the literature. Our result is therefore based on published data, but its accuracy is sensitive to some decisions that we were forced to take in the model construction phase. The first decision was to use survival estimates from the HF-HD arm of the Membrane Permeability Outcome ${ }^{16}$ randomized clinical trial. This trial was based on incident patients, whereas other studies on HDF were mainly performed on prevalent European dialysis patients. Additionally, the low crude mortality rate observed in the Membrane Permeability Outcome study may produce results not completely transferrable to ordinary (nontrial participating) patients. Indeed, as stressed by Palmer et al, ${ }^{20}$ relative risk reductions applied to low baseline risks produce low absolute reduction in event rates and low gains in health. It is possible that this low overall mortality rate affected the lack of difference between diabetic and nondiabetic patients. The reproducibility of the estimates remains therefore to be interpreted with caution.
The second point of discussion is the source of the effect estimators. As previously stressed, one of the main requirements for an economic evaluation is to include all the relevant evidence. With regard to the effectiveness data, this concept does not change, and meta-analysis is the usual technique employed to synthesize all the available evidence. To date five meta-analyses aggregating the results of the main convective dialysis therapy studies ${ }^{17,27-30}$ have been published. However, a huge heterogeneity of interventions was included in these meta-analyses, and the meta-analysis by Mostovaya et $\mathrm{al}^{17}$ was the only one which focused on trials on HDF as convective therapy with appropriate definition of convective volume. This was the basis for considering the related relative risk estimations in this model. Nevertheless, there is still a great variety of patients and treatment modalities characteristics among the studies. Indeed, for instance, both high- and low-flux modalities were included in the comparison. As a consequence, a relative risk based on a random effects model has been used, which makes the estimate of the pooled effect more conservative, and takes into account the clinical heterogeneity among the single studies. Of note, this meta-analysis includes also the CONTRAST study, and our cost-effectiveness analysis does include all the relevant randomized clinical trials. On the contrary, for instance, the economic evaluation by Mazairac is based only on the CONTRAST study; this is a subjective decision, while a complete cost-effectiveness analysis should instead take into consideration all the relevant available evidence, as previously pointed out.

With regard to the subanalysis of the diabetic patients, the ESHOL 5 estimates of risk reduction have been used. As the ESHOL study provided the most positive results in favor of HDF, concerns about the overestimation of the reduction in mortality could be raised. Nevertheless, the model did not show any difference between the two groups of patients, and this result highlights again that the impact on mortality is less important than the effect on the QoL.

Other limits for the current study have to be considered. The estimations used for this study were mainly derived from a meta-analysis with basically $2-3$ years follow-up time, but a more appropriate time horizon would be the patient's lifetime. This is especially true in the case of treatment of chronic diseases where the initiation of an intervention in middle-aged patients may have cost and effect implications on the rest of their lives. ${ }^{31}$ An important role of a decision model, therefore, is to bridge the gap between what has been observed in trials and what would be expected to happen over the long-term, forcing one to make assumptions about the long-term effects and consequences of treatment modalities. Additionally, the 
lack of QoL coefficients related to HF-HD forced the use of data from a comparison of HDF with low-flux HD. ${ }^{8}$

\section{Conclusion}

On the basis of this simulation HDF can be considered cost-effective. Uncertainty around results is high, but this is typical in this kind of analyses: neglecting some available evidence only because of its uncertainty would contrast with the comprehensive nature of an economic model. This study contributes also to show the scarcity and heterogeneity of data available in the literature that jeopardize the economic evaluation of HDF when compared to HF-HD. New studies are needed to assess more precisely the differential benefits between the therapies: value-of-information analysis might help to identify more specifically the parameters which would be worth to be further investigated. A more comprehensive evaluation performed in a randomized clinical trial is required in order to include other relevant cost components (eg, pharmaceuticals, hospitalizations, prevention of longterm dialysis-related complications, beta- 2 microglobulinamyloidosis, and transplantation access) in the analysis. Possible savings resulting from a reduction in the cost of ancillary pharmacological therapy and hospitalization have not been considered in the current analysis.

\section{Disclosure}

$\mathrm{DM}$ and $\mathrm{BC}$ are employees of Fresenius Medical Care. The authors report no other conflicts of interest in this work.

\section{References}

1. Sichart JM, Moeller S. Utilization of hemodiafiltration as treatment modality in renal replacement therapy for end-stage renal disease patients - a global perspective. Contrib Nephrol. 2011;175: 163-169.

2. Canaud B, Bragg-Gresham JL, Marshall MR, et al. Mortality risk for patients receiving hemodiafiltration versus hemodialysis: European results from the DOPPS. Kidney Int. 2006;69(11):2087-2093.

3. Canaud B, Bayh I, Marcelli D, et al. Improved survival of incident patients with high-volume haemodiafiltration: a propensity-matched cohort study with inverse probability of censoring weighting. Nephron. 2015;129(3):179-188.

4. Akizawa T, Koiwa F. Clinical expectation of online hemodiafiltration: a Japanese perspective. Blood Purif. 2015;40(Suppl 1):12-16.

5. Maduell F, Moreso F, Pons M, et al. High-efficiency postdilution online hemodiafiltration reduces all-cause mortality in hemodialysis patients. J Am Soc Nephrol. 2013;24(3):487-497.

6. Ok E, Asci G, Toz H, et al; Turkish Online Haemodiafiltration Study. Mortality and cardiovascular events in online haemodiafiltration (OLHDF) compared with high-flux dialysis: results from the Turkish OLHDF study. Nephrol Dial Transplant. 2013;28(1):192-202.

7. Grooteman MP, van den Dorpel MA, Bots ML, et al; CONTRAST Investigators. Effect of online hemodiafiltration on all-cause mortality and cardiovascular outcomes. J Am Soc Nephrol. 2012;23(6): 1087-1096.
8. Mazairac AH, Blankestijn PJ, Grooteman MP, et al; CONTRAST Investigators. The cost-utility of haemodiafiltration versus haemodialysis in the Convective Transport Study. Nephrol Dial Transplant. 2013;28(7):1865-1873.

9. Oates T, Cross J, Davenport A. Cost comparison of online haemodiafiltration with high-flux haemodialysis. J Nephrol. 2012;25(2):192-197.

10. Vanholder R, Van Biesen W, Lameire N. Renal replacement therapy: how can we contain the costs? Lancet. 2014;383(9931):1783-1785.

11. Bolasco $\mathrm{P}$, Contu A, Meloni $\mathrm{P}$, Vacca D, Galfrè A. Microbiological surveillance and state of the art technological strategies for the prevention of dialysis water pollution. Int $J$ Environ Res Public Health. 2012;9(8):2758-2771.

12. Lebourg L, Amato S, Toledano D, Petitclerc T, Créput C. [Online hemodiafiltration: is it really more expensive?]. Nephrol Ther. 2013;9(4):209-214. French.

13. Briggs A, Sculpher M, Dawson J, Fitzpatrick R, Murray D, Malchau $\mathrm{H}$. The use of probabilistic decision models in technology assessment: the case of total hip replacement. Appl Health Econ Health Policy. 2004;3(2):79-89.

14. Briggs A, Sculpher M, Claxton K. Decision Modelling for Health Economic Evaluation. Oxford, UK: Oxford University Press; 2006.

15. Drummond MF. Methods for the Economic Evaluation of Health Care Programmes. Oxford, UK: Oxford University Press; 2005.

16. Locatelli F, Martin-Malo A, Hannedouche T, et al; Membrane Permeability Outcome (MPO) Study Group. Effect of membrane permeability on survival of hemodialysis patients. J Am Soc Nephrol. 2009;20(3): 645-654.

17. Mostovaya IM, Blankestijn PJ, Bots ML, et al; EUDIAL1 - an official ERA-EDTA Working Group. Clinical evidence on hemodiafiltration: a systematic review and a meta-analysis. Semin Dial. 2014;27(2):119-127.

18. Del Vecchio L, Giordana G, Pedrini L, Marcelli D, Sisti N. Elements for economic evaluation on online hemofiltration (ol-HDF) versus standard haemodialysis to treat patients with end-stage renal disease (ESRD). Ital J Public Health. 2012;9(4 (Suppl 1)):S1-S51.

19. National Institute for Health and Care Excellence. Guide to the Methods and Technology Appraisal 2013. London: National Institute for Health and Care Excellence; 2013.

20. Palmer S, Sculpher M, Philips Z, et al. Management of non-ST-elevation acute coronary syndromes: how cost-effective are glycoprotein IIb/IIIA antagonists in the UK National Health Service? Int J Cardiol. 2005; 100(2):229-240.

21. Briggs AH, Goeree R, Blackhouse G, O’Brien BJ. Probabilistic analysis of cost-effectiveness models: choosing between treatment strategies for gastroesophageal reflux disease. Med Decis Making. 2002;22(4):290-308.

22. Griffin S, Claxton K, Hawkins N, Sculpher M. Probabilistic analysis and computationally expensive models: necessary and required? Value Health. 2006;9(4):244-252.

23. van de Luijtgaarden MWM, Jager KJ, Segelmark M, et al. Trends in dialysis modality choice and related patient survival in the ERA-EDTA Registry over a 20-year period. Nephrol Dial Transplant. 2016;31(1): $120-128$.

24. Lévesque R, Marcelli D, Cardinal H, et al. Cost-effectiveness analysis of high-efficiency hemodiafiltration versus low-flux hemodialysis based on the Canadian Arm of the CONTRAST Study. Appl Health Econ Health Policy. 2015;13(6):647-659.

25. Mennini FS, Russo S, Marcellusi A, Quintaliani G, Fouque D. Economic effects of treatment of chronic kidney disease with low-protein diet. J Ren Nutr. 2014;24(5):313-321.

26. McBrien KA, Manns BJ. Haemodiafiltration: not effective or costeffective compared with haemodialysis. Nephrol Dial Transplant. 2013;28(7):1630-1633; discussion 1633.

27. Rabindranath KS, Strippoli GF, Roderick P, Wallace SA, MacLeod AM, Daly C. Comparison of hemodialysis, hemofiltration, and acetate-free biofiltration for ESRD: systematic review. Am J Kidney Dis. 2005; 45(3):437-447. 
28. Susantitaphong P, Siribamrungwong M, Jaber BL. Convective therapies versus low-flux hemodialysis for chronic kidney failure: a meta-analysis of randomized controlled trials. Nephrol Dial Transplant. 2013;28(11):2859-2874.

29. Wang AY, Ninomiya T, Al-Kahwa A, Perkovic V, Gallagher MP, Hawley C, Jardine MJ. Effect of hemodiafiltration or hemofiltration compared with hemodialysis on mortality and cardiovascular disease in chronic kidney failure: a systematic review and meta-analysis of randomized trials. Am J Kidney Dis. 2014;63(6):968-978.
30. Nistor I, Palmer SC, Craig JC, Saglimbene V, Vecchio M, Covic A, Strippoli GF. Convective versus diffusive dialysis therapies for chronic kidney failure: an updated systematic review of randomized controlled trials. Am J Kidney Dis. 2014;63(6):954-967.

31. Kobelt G, Jönsson L, Young A, Eberhardt K. The cost-effectiveness of infliximab (Remicade) in the treatment of rheumatoid arthritis in Sweden and the United Kingdom based on the ATTRACT study. Rheumatology (Oxford). 2003;42(2):326-335.

\section{Publish your work in this journal}

ClinicoEconomics and Outcomes Research is an international, peerreviewed open-access journal focusing on health technology assessment, pharmacoeconomics and outcomes research in the areas of diagnosis, medical devices, and clinical, surgical and pharmacological intervention. The economic impact of health policy and health systems
Submit your manuscript here: https://www.dovepress.com/clinicoeconomics-and-outcomes-research-jorth

organization also constitute important areas of coverage. The manuscript management system is completely online and includes a very quick and fair peer-review system, which is all easy to use. Visit http://www.dovepress.com/testimonials.php to read real quotes from published authors. 